\title{
AUTOMATED ViSUAL INSPECTION SYSTEM IN PRALINE INDUSTRY
}

\author{
Irina Topalova
}
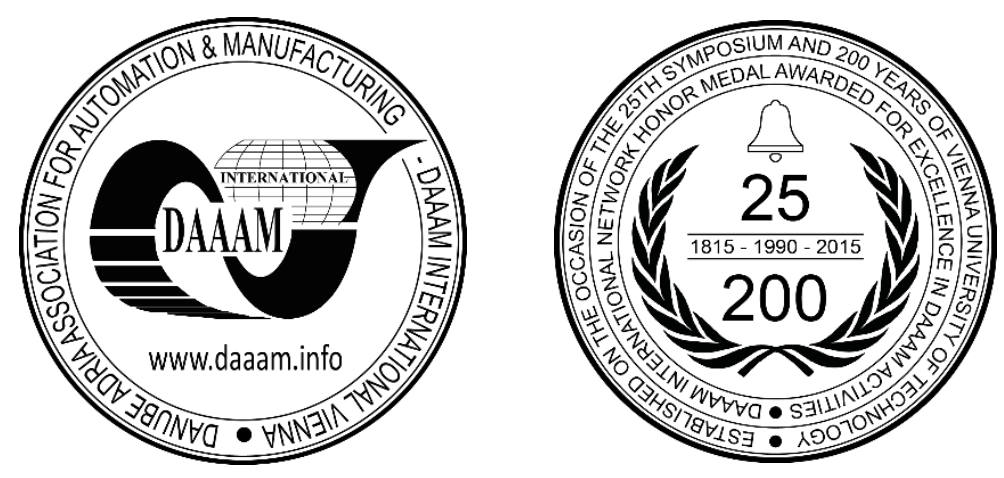

This Publication has to be referred as: Topalova, I[rina] (2016). Automated Visual Inspection System in Praline Industry, Proceedings of the 27th DAAAM International Symposium, pp.0175-0179, B. Katalinic (Ed.), Published by DAAAM International, ISBN 978-3-902734-08-2, ISSN 1726-9679, Vienna, Austria

DOI: $10.2507 / 27$ th.daaam.proceedings.026

\begin{abstract}
There are many industrial applications of sophisticated food packaging systems developed nowadays. An essential part of these systems is the visual inspection of the package quality. One of the most important tasks in the automated food packaging is the determination of missing elements in the package. The major drawback of these systems is the implementation of too complex techniques, hardware and software methods which raises the cost of the automated system. This paper represents a hardware, software method and algorithm solution for determining missing pralines when automatically loading them into the trays. The main objectives of the research are focused on developing a simple visual method and algorithm using standard hardware components and communication interfaces, easily compatible with different control systems. An image histogram correlation determination is applied on the off-line programing stage. Based on the results, the system is trained with a simple parametrical vector including different histogram parameters to define the number of missing pralines in the tray. The system was tested with different blurred tray images to simulate the movement of the conveyer belt. The results of the obtained high classification accuracy and fast response are represented and discussed. The opportunities for further development of the system are also presented.
\end{abstract}

Keywords: Automated system; Visual inspection; Histogram; Praline packaging.

\section{Introduction}

There are many industrial applications of automated food packaging systems developed nowadays, that implement visual inspection of the package quality. But the major disadvantage of these systems is the implementation of too complex techniques, hardware and software methods which makes the automated system too expensive. One of the most important tasks in the automated food packaging is the determination of missing parts in the package. There are many automated packaging systems in praline industry, but they are too complex or not developed for checking of missing pralines. For example, Mecmesin Corporation [1] produces comprehensive range of standard package testing machines but each user defined case, demands development and adjustment of specific equipment. They apply an autonomous mechanical system also for checking the presence of pills in a blister. Gerhard Schubert GmbH [2] offers an excellent praline packaging automated line with a visual system for recognition of praline type or damaged exemplars, before filling the trays. But the system does not include checking for missing pralines, after automated arranging the tray. AMP Rose Chocolate investor [3] supplies chocolate processing machinery lines to suit customer requirements with no package control. Some systems developed by Mettler Toledo [4] offer detection of missing food parts applying X-Rey inspection, 
which acquires additional equipment or sometimes causes food spoilage. The authors in [5] use support vector machines to analyze the texture feature vector of fruits in order to classify them in groups. Analyzing the hue plane of an image, every pixel is described with the help of its feature vector by analysis a small neighborhood of $7 \times 7$ pixels, with the current pixels as a center. For each pixel a 6-value-vector is constructed, including a mean value, standard deviation, smoothness, third moment, uniformity and entropy. In [6] the authors calculate the area, perimeter, shape, roundness und apply a fuzzy logic method for automatic analysis of X-ray images of industrial products for defect detection. Both methods use too much complex preprocessing calculations, constructing unnecessary detailed feature vector. This causes increase in computational time. To overcome these disadvantages a simple method, algorithm and visual inspection system is proposed. It uses standard hardware components and communication interfaces, easily compatible with different control systems. An image histogram correlation determination is applied on the off-line programing stage. Based on the results, the system is trained with a simple parametrical vector including different histogram parameters to define the number of missing pralines in the tray. The system was tested with different blurred tray images to simulate the movement of the conveyer belt. The results of the obtained high classification accuracy and fast response are represented and discussed. The opportunities for further development of the system are also presented.

\section{Proposed visual inspection system}

The proposed visual inspection system consists of a conventional smart camera with triggered infrared lighting and a Vision Builder software package for development of user defined visual inspection algorithms. Different integrated standard interface drivers give good opportunity for further connection with any real-time working control system. The developed algorithm consists of a pre-processing stage for image histogram extraction, correlation analysis between images with different number of missing pralines, histogram reduction and extraction of histogram parameters. The extracted reduced histogram parameters form train parametrical vectors for a minimum mean distance classifier. Different blurred tray images were used for testing purpose to simulate the image acquisition by different conveyer belt velocities. The achieved high classification accuracy, fast response and standard interface compatibility turn it into a reliable and easy to use automated system.

\subsection{Proposed algorithm}

The initial step of the algorithm includes image acquisition and histogram extraction. Four classes were investigated

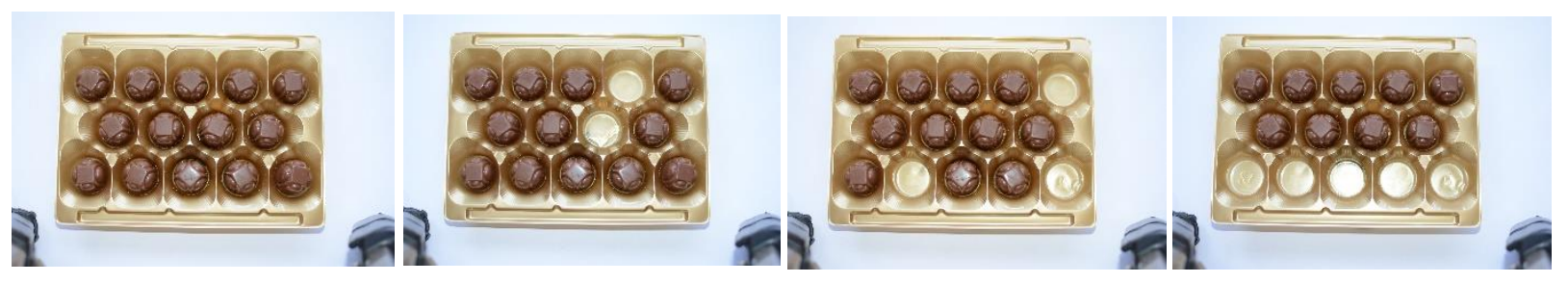

Fig. 1. Exemplars of the four investigated classes - full, miss-2, miss-3, miss-5

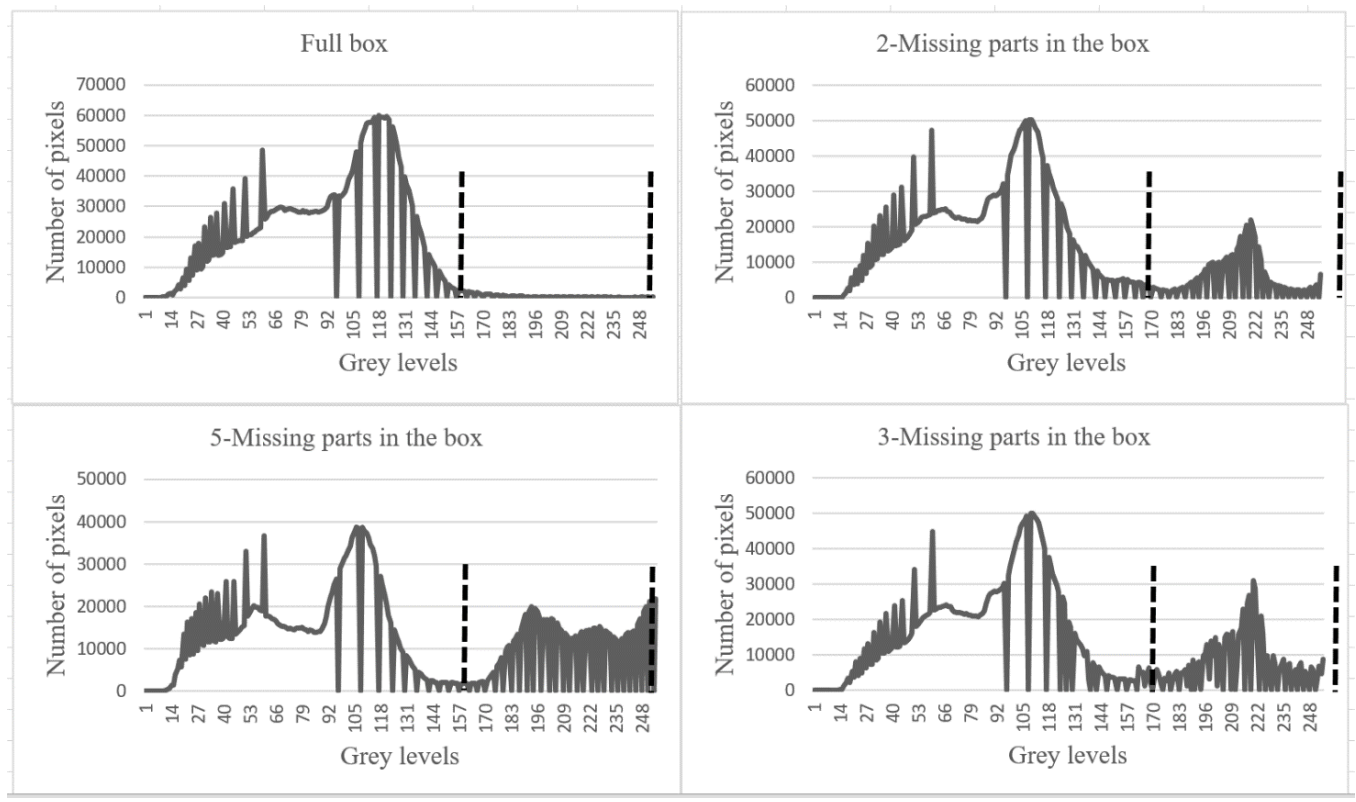

Fig. 2. Grey level histograms of the four classes - full, miss-2, miss-3, miss-5 
with a full tray, with missing 2, 3 or 5 pralines having corresponding names full, miss-2, miss-3, miss-5, shown in Fig.1. - Next the grey level histograms were calculated on the basis of calculated mean RGB value. The histograms are calculated only over Region of Interest (ROI), fixed on the praline nests in the tray. To obtain invariance against rotation and translation of the tray, the ROIs are related to a coordinate system, which is fixed to a predefined pattern. This pattern is found after scanning the image and searching the highest value of the cross-correlation coefficient $C(i, j)$ between the chosen pattern and the image

$$
C(i, j)=\sum_{x=0}^{L-1} \sum_{y=0}^{K-1} t(x, y) f(x+i, y+j),
$$

where $i=0,1, \ldots N-1, j=0,1, \ldots M-1$, where $t(x, y)$ is the pattern, $M$ and $N$ are the image dimensions, $\mathrm{L}$ and $\mathrm{K}$ are pattern dimensions. The partial cross-correlation [7] between each two histograms moving the sliding window with a step of five grey levels is calculated. The highest value of the cross-correlation coefficient varies between 0.78 and 0.89 for the range between 0 and 160 grey level and the lowest values between -0.09 and 0.02 for the range between 0 and 160 grey levels. This means the lower grey level values of all histograms are high overlapping, while the range between 160 and 255 grey level values are much more distinguishable.

- As next step all histograms are reduced taking only the low correlated ranges between 160 and 255 grey levels. Only four parameters of the reduced histograms are chosen to form the parametrical vector, used to train the classifier. These parameters are [8]: mean histogram value (mean), standard deviation (stdev), median value (median) and histogram skew (skew). For each class $j$ a mean parametrical vector $\overline{\boldsymbol{M}}_{j}$ is calculated over 10 exemplars.

$$
\overline{\mathbf{M}}_{j}=\left\{\overline{\operatorname{mean}}^{j}, \overline{\operatorname{stdev}}^{j}, \overline{\operatorname{median}}^{j}, \overline{\text { skew }}^{j}\right\}
$$

- The calculated parametrical vector for each current image $\boldsymbol{X}_{i}=\left\{X_{1}^{i}, X_{2}^{i}, \ldots, X_{n_{i}}^{i}\right\}$, (where in our case $n_{i}=4$ ), is classified applying minimum mean distance classifier. It means $\boldsymbol{X}_{i}$ belongs to class $C j$ if (3) is true.

$$
\boldsymbol{X}_{i} \in \operatorname{Class} C_{j}, \text { if } d\left(\boldsymbol{X}, \overline{\boldsymbol{M}}_{j}\right)=\min d\left(\boldsymbol{X}, \overline{\boldsymbol{M}}_{j}\right)
$$

where for $d$ Manhattan distance is chosen.

- In the last step, the current calculated vector parameters and the recognized class are visualized. The results are prepared for export through standard interfaces.

\subsection{System development}

The experiments are implemented using a Smart Camera NI 1742 with triggered infrared lighting and software Vision Builder AI 2014. The constructed vision inspection system is shown in Fig.3. To simulate the blur noise [8] added to the images because of the conveyer belt moving, the calculated blur in number of pixels is added to each image. For a typical conveyer belt velocity of $25 \mathrm{~m} / \mathrm{min} 417 \mathrm{~mm} / \mathrm{sec}$ and image resolution of 300 dpi $118,11 \mathrm{dp}(\mathrm{cm}) 11,81 \mathrm{dp}(\mathrm{mm})$, the calculated conveyer belt velocity measured in Pixel per second is $\mathrm{Vp}=417 \mathrm{x} 11,8=4920 \mathrm{Pix} / \mathrm{sec}$. The resulting Motion Blur $=(\mathrm{Vp}) \times($ Exposure time $)=4920 \times 1 / 12540$ Pix Motion Blur. In our case, a short value of Exposure time $=1 / 500$ is chosen which corresponds to 9 Pix Motion Blur.

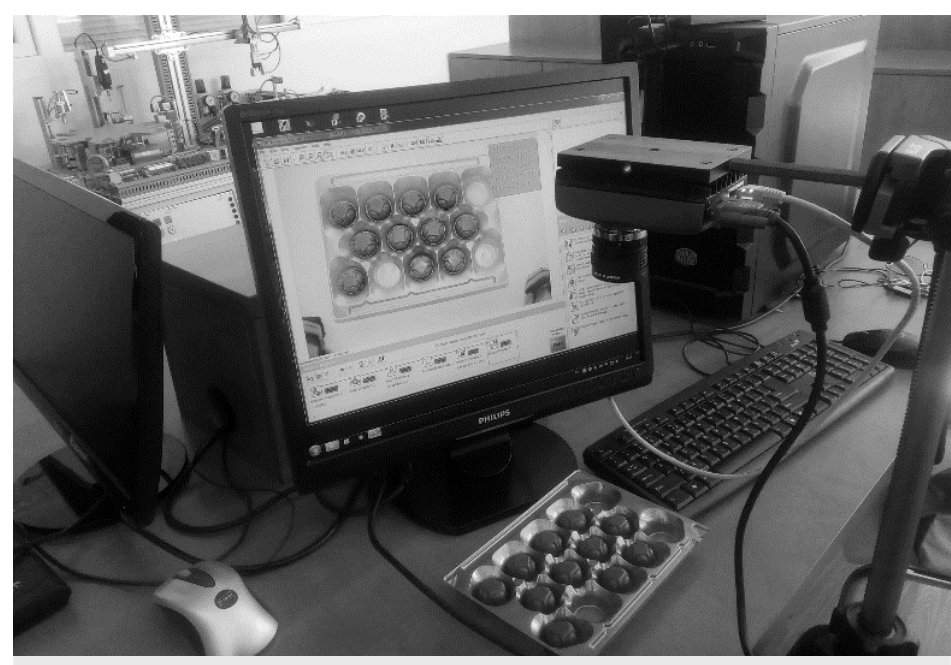

Fig. 3. The constructed vision inspection system 


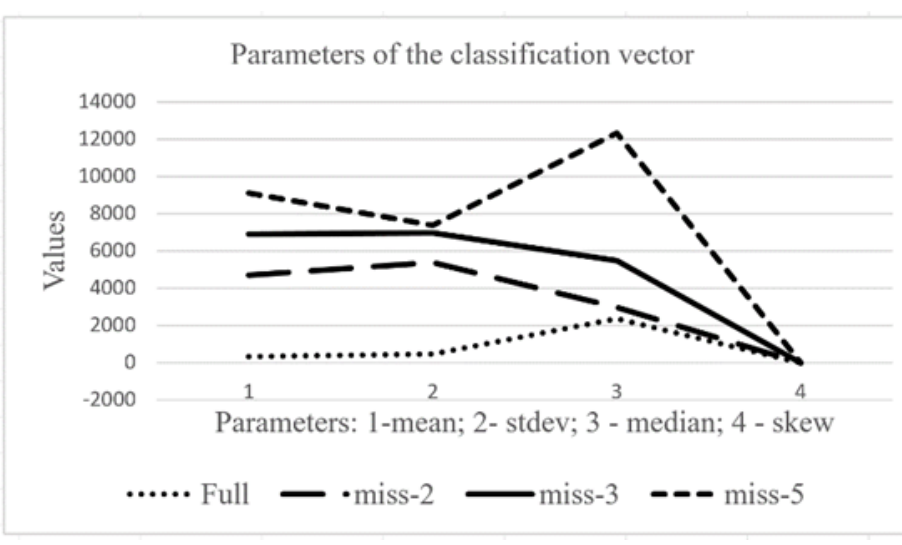

a
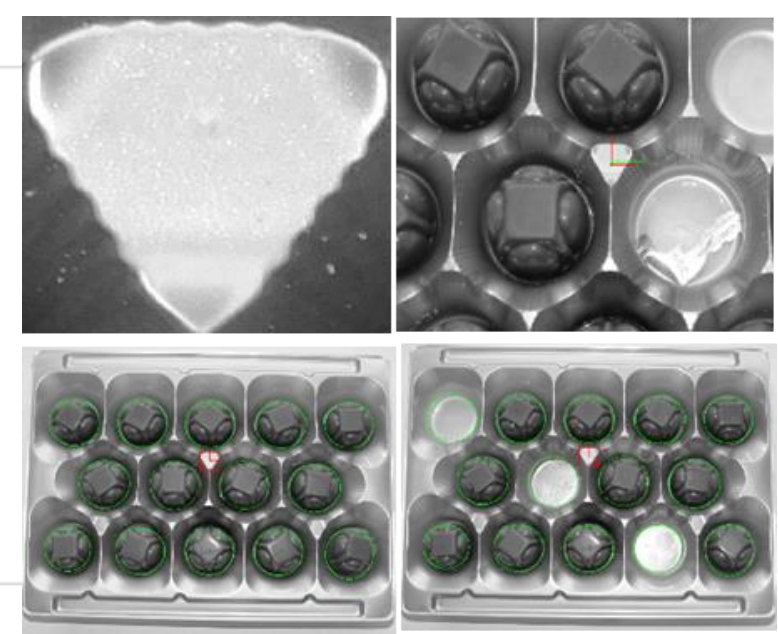

b

Fig. 4. a/ Parameters of the classification vector $\overline{\boldsymbol{M}}_{j}$ for classes full, miss-2, miss-3, miss-5 $b /$ Pattern determination, fixed coordinate system and ROIs

This value is added to some of the test images [9]. Fig.4 a/ shows the obtained parameters of the classification vector $\overline{\boldsymbol{M}}_{j}$ for classes full, miss-2, miss-3, miss-5 calculated with mean values of each parameter calculated over 10 different images. It is used as support/reference vector for the calculations in (3). Fig.4 b/ represents the determination of chosen Pattern, fixed with the pattern coordinate system and the ROIs used for histogram calculations. The given images represent slightly rotated trays. The corresponding steps in the algorithm are named as follows: Image Acquisition, Image Improvement with histogram calculation and reduction, Match Pattern, Set Coordinate System, Parameter calculation and Display of the results, data preparation for export through standard interfaces.

\section{Experimental results}

The system functionality was tested with 20 exemplars/images of each class. To some of them Motion Blur of 9Pixels, of 15Pixels and even of 25 Pixels was added as in [10]. Fig.5 shows the distribution of parameters median, mean and stdev for 20 exemplars of classes: miss-2, miss-3 and miss-5 respectively. Obviously there are some exemplars overlapping in the parametrical feature space. The proposed method and algorithm separates them very good at the system

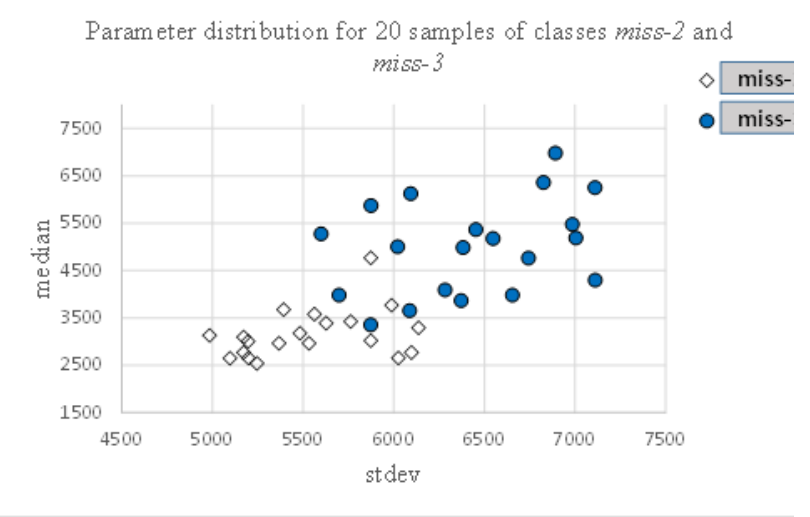

a

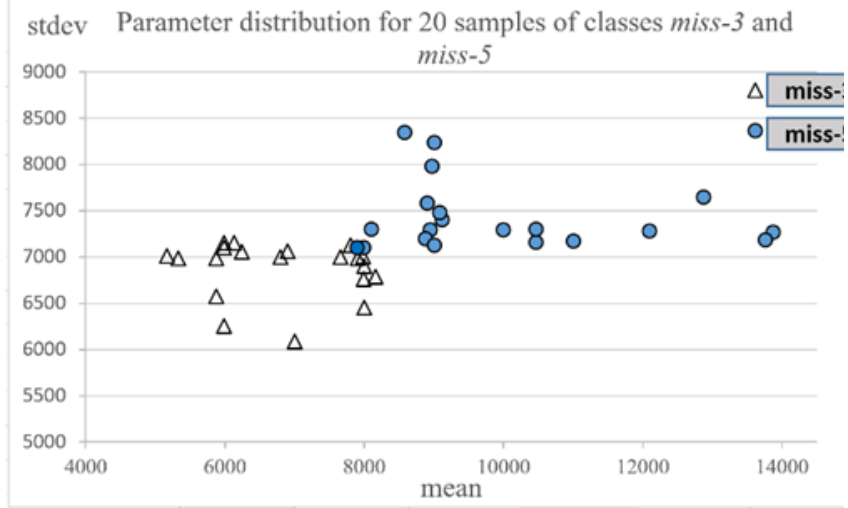

b

Fig. 5. a/ Distribution of parameters median and stdev for 20 exemplars of classes miss-2 and miss-3; b/ Distribution of parameters mean and stdev for 20 exemplars of classes miss-3 and miss-5

output, because the obtained classification accuracy is $100 \%$. The accuracy is calculated as [(number of all exemplars number of exemplars with bad line fitting)/ number of all exemplars].100 [\%]. Fig. 6 shows the output of the developed user program display when recognizing class miss-2. Table 1 represents State/Step elapsed times in $n s$ based on 100 inspection run(s). The very short export time of $1.1186 \mathrm{~ms}$ ensures the possibility of using all available standard export interfaces in Vision Builder AI 2014. They are Serial I/O, Pulse I/O, Modbus, TCP/I/O, Ethernet/IP [11]. The obtained short Total execution time gives opportunity for application in real-time control systems. 


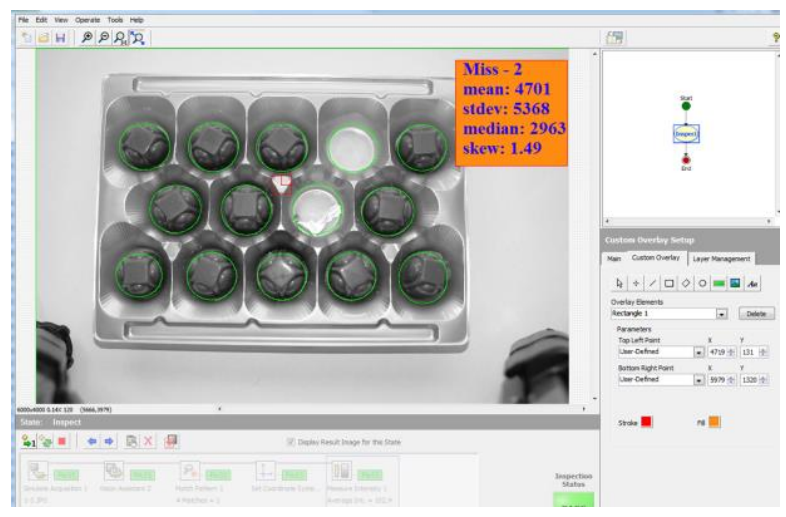

Fig. 6 Output of the developed user program display when recognizing class miss-2

\begin{tabular}{|c|c|c|c|c|c|c|}
\hline Step Name & Iterations & Total Time & Average & Std-Dev & Shortest & Longest \\
\hline Inspect & 100 & 188066.277 & 1880.663 & 25.604 & 1840.41 & 2041.688 \\
\hline Image Acquisition & 100 & 46291 & 462.91 & 4.557 & 443 & 473 \\
\hline Image Improvement & 100 & 19549 & 195.49 & 2.335 & 189 & 204 \\
\hline Match Pattern & 100 & 62413.682 & 624.137 & 20.595 & 591.062 & 760.59 \\
\hline Set Coordinate System & 100 & 35.562 & 0.356 & 0.044 & 0.252 & 0.453 \\
\hline Parameter calculation & 100 & 58486.004 & 584.86 & 9.589 & 566.288 & 620.191 \\
\hline Display/data export & 100 & 1185.75 & 11.857 & 3.614 & 9.681 & 40.702 \\
\hline
\end{tabular}

Table 1. State/Step elapsed times in $n s$ based on 100 inspection run(s).

\section{Conclusion}

The developed inspection system offers high classification accuracy, beside simple technical equipment. Applying the proposed calculations only over the reduced histograms and only for the defined ROIs contributes to fast computational response. Using a relevant coordinate system, fixed to a chosen pattern enables a recognition invariant against image rotation and translation. The system is easy-coupled to various control devices because of data export to different interfaces. The achieved high classification accuracy, fast response and standard interface compatibility turn it into a reliable and easy to use automated system. In the future a connection to a National Instruments real-time working controll system is forseen. Also as future work different tests with normalized histograms is planed, intending to make the system invariant against variations in the image intensity.

\section{References}

[1] http://www.mecmesin.com/flexible-packaging-welded-seal-strength-cs6, Accessed on: 2016-08-15

[2] http://www.gerhard-schubert.de/de/verpackungsmaschinen-aus-crailsheim, Accessed on: 2016-08-10

[3] http://www.amp-rose.com/used-machines/chocolate/_Accessed on: 2016-08-08

[4] http://www.mt.com/int/en/home/applications/Product-Inspection_2/PI-ProductInte.html_Accessed on: 2016-08-11

[5] Rakun, J.; Berk, P. \& Lakota, M. (2015). Fruit Sorting Based on Texture Analysis and Support Vector Machine Classification, Chapter 19 in DAAAM International Scientific Book 2015, B. Katalinic (Ed.), Published by DAAAM International, pp. 209-218, ISBN 978-3-902734-05-1, ISSN 1726-9687, Vienna, Austria,

DOI: 10.2507/daaam.scibook.2015.19.

[6] Amza, C. G. \& Cicic. D. T. (2014) Industrial Image Processing Using Fuzzy-Logic, Proceedings of 25th DAAAM International Symposium on Intelligent Manufacturing and Automation, Vol. 100-2015, ISSN 1877-DAAAM 2014 7058, Katalinic, B. (Ed.), pp. $492-498$.

[7] Leydesdorff, L. \& Vaughan, L. (2006). Co-occurrence matrices and their applications in information science: extending ACA to the Web environment. Journal of the American Society for Information Science and Technology 57(12), 1616-1628.

[8] Gonzalez, R., C. \& Woods, R., E. (2002) Digital image processing, 2nd ed. Boston, MA, USA: Prentice-Hall of India, 2002.

[9] B. G. Batchelor, B., G. (2012) Machine Vision Handbook, Springer, ISBN 9781849961684, 2012.

[10] Topalova, I., (2015) Method for Parameter Adjustment for Automated Visual Inspection of Bottled Liquids, IARIA/ ICAS'2015 - 9th International Conference on Autonomic and Autonomous Systems, May, pp. 16-19, ISBN: 978161208-405-3, Rome, Italy, 2015.

[11] National Instruments, (2009) NI Vision Builder for Automated Inspection User Manual, 373379C-0. National Instruments, 2009. 